\title{
ARTICLE
}

Received 13 Nov 2012 | Accepted 6 Feb 2013 | Published 5 Mar $2013 \quad$ DOl: 10.1038/ncomms2578

\section{Fractional Bloch oscillations in photonic lattices}

\author{
Giacomo Corrielli ${ }^{1,2}$, Andrea Crespi ${ }^{1,2}$, Giuseppe Della Valle ${ }^{1,2}$, Stefano Longhi ${ }^{1,2} \&$ Roberto Osellame ${ }^{1,2}$
}

Bloch oscillations, the oscillatory motion of a quantum particle in a periodic potential, are one of the most fascinating effects of coherent quantum transport. Originally studied in the context of electrons in crystals, Bloch oscillations manifest the wave nature of matter and are found in a wide variety of different physical systems. Here we report on the first experimental observation of fractional Bloch oscillations, using a photonic lattice as a model system of a two-particle extended Bose-Hubbard Hamiltonian. In our photonic simulator, the dynamics of two correlated particles hopping on a one-dimensional lattice is mapped into the motion of a single particle in a two-dimensional lattice with engineered defects and mimicked by light transport in a square waveguide lattice with a bent axis.

\footnotetext{
${ }^{1}$ Istituto di Fotonica e Nanotecnologie - Consiglio Nazionale delle Ricerche, Piazza Leonardo da Vinci, 32, I-20133 Milano, Italy. ${ }^{2}$ Dipartimento di Fisica Politecnico di Milano, Piazza Leonardo da Vinci, 32, I-20133 Milano, Italy. Correspondence and requests for materials should be addressed to R.O. (email: roberto.osellame@ifn.cnr.it).
} 
B loch oscillations (BO), that is, the oscillatory motion of a quantum particle in a periodic potential driven by a constant force, constitute one of the most striking and oldest predictions of coherent quantum transport in periodic lattices ${ }^{1,2}$. In natural crystals, BO have never been observed because of dephasing effects. Solely with the advent of semiconductor superlattices and ultracold atoms, BO have been observed for matter waves ${ }^{3-6}$. In their essence, $\mathrm{BO}$ are a wave phenomenon. As such, they are found for optical ${ }^{7-11}$ and acoustic $^{12}$ waves as well. When interactions between particles compete with their mobility, novel dynamical behaviour can arise where particles form bound states ${ }^{13}$ and co-tunnel through the lattice ${ }^{14}$. While particle interaction has been generally associated to BO damping ${ }^{15-17}$, for few strongly interacting particles it was predicted that bound states undergo fractional $\mathrm{BO}$ at a frequency twice (or multiple) that of single-particle $\mathrm{BO}^{18-20}$. The observation of fractional $\mathrm{BO}$ is challenging in condensed-matter systems and up to now has not been achieved even in model systems.

Ultracold quantum gases in optical lattices have provided in the past decade a powerful laboratory tool to simulate Hubbard models of condensed-matter physics ${ }^{21}$. Experiments with ultracold atoms have so far demonstrated the existence of bound-particle states and correlated tunnelling phenomena ${ }^{13,14}$, however fractional $\mathrm{BO}$ of atomic pairs have not been observed yet. A photonic simulator of the Hubbard model can map the dynamics of two correlated particles hopping on a onedimensional (1D) lattice into the motion of a single particle in a $2 \mathrm{D}$ lattice with engineered defects ${ }^{22,23}$, thereby offering the possibility to easily visualize the effect under simulation, but requiring a high level of control of the photonic structure. Here we report on the first observation of fractional $\mathrm{BO}$ using a photonic lattice as a model system of a few-particle extended Bose-Hubbard Hamiltonian, which is the most appropriate theoretical framework to consistently describe fractional BO.

\section{Results}

Theory of BO in the extended Bose-Hubbard (EBH) model. To study correlated $\mathrm{BO}$, we consider an EBH model ${ }^{24-26}$ describing strongly interacting bosons in the lowest Bloch band of a $1 \mathrm{D}$ lattice driven by an external force. This model is more accurate than the standard $\mathrm{BH}$ model as it accounts for higher-order processes whose magnitude is comparable with the one of second-order tunnelling ${ }^{26}$. The Hamiltonian of the system is given by $\hat{H}=\hat{H}_{\mathrm{EBH}}+\hat{H}_{F}$, where $\hat{H}_{F}=F d \sum_{l} l \hat{n}_{l}$ describes the effect of the external constant force $F$ ( $d$ is the lattice period) and $\hat{H}_{\mathrm{EBH}}=\hat{H}_{\mathrm{BH}}+\hat{H}_{3}+\hat{H}_{4}+\hat{H}_{5}$ is the EBH Hamiltonian. $\hat{H}_{\mathrm{EBH}}$ includes the standard BH Hamiltonian (with $\hbar=1$ )

$$
\hat{H}_{\mathrm{BH}}=\frac{U_{0}}{2} \sum_{l} \hat{n}_{l}\left(\hat{n}_{l}-1\right)-\frac{J}{2} \varepsilon \sum_{l}\left(\hat{a}_{l}^{\dagger} \hat{a}_{l+1}+\text { H.c. }\right)
$$

and three additional terms ${ }^{24}$, whose explicit expression is given in Supplementary Note 1 . In equation (1), $U_{0}$ is the on-site energy interaction strength $\left(U_{0}>0\right.$ for a repulsive interaction); $J$ is related to the single-particle hopping rate between adjacent lattice sites; $\varepsilon \ll 1$ is the lattice attenuation factor ${ }^{24}$; $\hat{a}_{l}^{\dagger}$ and $\hat{a}_{l}$ are the bosonic creation and annihilation operators and $\hat{n}_{l}=\hat{a}_{l}^{\dagger} \hat{a}_{l}$ is the particle number operator at lattice site $l$. The standard $\mathrm{BH}$ term $\hat{H}_{\mathrm{BH}}$ describes on-site particle-particle interaction and singleparticle tunnelling between adjacent lattice sites (Fig. 1a, b). The additional terms $\hat{H}_{3}, \hat{H}_{4}$ and $\hat{H}_{5}$ account for nearest-neighbor hopping of single atoms conditioned by the on-site occupation number, nearest-neighbor interactions, and nearest-neighbor hopping of bosonic pairs, respectively (Fig. 1c,d). a

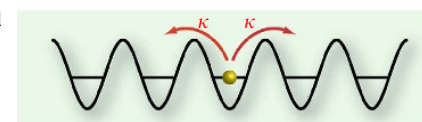

b

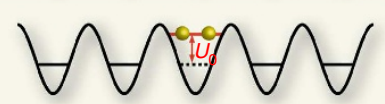

C

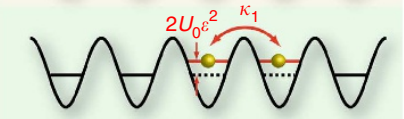

d

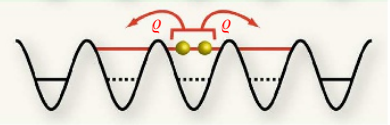

e

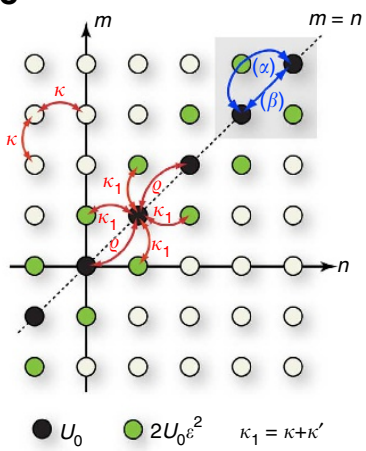

Figure 1 | Dynamics of two interacting bosons in the extended Bose-Hubbard lattice. Two-particle dynamics in the EBH model: (a) Single-particle tunnelling; (b) on-site particle interaction;

(c) nearest-neighbor-site particle interaction and conditional single-particle tunnelling; (d) nearest-neighbor hopping of particle pairs. (e) Fock-space representation of the two-particle dynamics; the upper-right inset represents the two possible pathways for two-particle hopping to the nearest-neighbor site: $(\alpha)$ is the second-order pair tunnelling, while $(\beta)$ is the direct two-particle tunnelling.

In the absence of particle interaction, each particle undergoes independent $\mathrm{BO}$ on the lattice. The single-particle amplitude probabilities $A_{n}(t)$ to find the particle at lattice site $n$ evolve according to the coupled equations

$$
i \frac{d A_{n}}{d t}=-\kappa\left(A_{n+1}+A_{n-1}\right)+F d n A_{n}
$$

where $\kappa=\varepsilon J / 2$ is the single-particle hopping rate. The singleparticle energy spectrum is given by an equally spaced WannierStark ladder ${ }^{27,28}$, which is the spectral counterpart of $\mathrm{BO}$ and results in a periodic dynamics at the frequency $\omega_{\mathrm{B}}=F d$. For single-site excitation, that is, $A_{n}(0)=\delta_{n, 0}, \mathrm{BO}$ appear as a breathing (rather than an oscillatory) motion at the frequency $\omega_{\mathrm{B}}$. Photonic demonstrations of such $\mathrm{BO}$ breathing dynamics were previously reported in refs $7,8,10$.

Let us now consider the dynamics of two interacting particles. The state vector $|\psi(t)\rangle$ of the system can be expanded in Fock space as $|\psi(t)\rangle=(1 / \sqrt{2}) \sum_{n, m} c_{n, m}(t) \hat{a}_{n}^{\dagger} \hat{a}_{m}^{\dagger}|0\rangle$, where $c_{n, m}(t)$ is the amplitude probability to find one particle at site $n$ and the other particle at site $m$, with $c_{n, m}=c_{m, n}$ for bosons. The evolution equations for the amplitude probabilities (Supplementary Note 2) show that the dynamics of the two correlated bosons on the 1D lattice can be mapped into the motion of a single-particle hopping on the 2D square lattice of Fig. 1e. The square lattice shows both energy site defects and hopping rate corrections on the three diagonals $m=n, n \pm 1$. Far from the main diagonal, the square lattice is homogeneous with a uniform hopping rate $\kappa=\varepsilon J / 2$, which is the single-particle hopping rate of the original problem (Fig. 1a). The site energy defects $U_{0}$ and $2 \varepsilon^{2} U_{0}$ on the main $(m=n)$ and nearest-neighbor $(m=n \pm 1)$ diagonals account for on-site and nearest-neighbor-site particle repulsion, respectively (Fig. 1b,c). The correction of hopping rates $\kappa_{1}=\kappa+\kappa^{\prime}$ (with $\kappa^{\prime}=-U_{0} \varepsilon^{3 / 2}$ ) on the main diagonal accounts for nearestneighbor hopping of single atoms conditioned by the on-site occupation number (Fig. 1c). Finally, the cross coupling $\rho=-2 U_{0} \varepsilon^{2}$ on the main diagonal $n=m$ describes nearestneighbor hopping of bosonic pairs, that is, direct two-particle tunnelling (Fig. 1d). These additional terms could degrade BO and therefore their effect has been carefully investigated (Supplementary Note 2). It can be shown that for two strongly 
correlated particles $\left(J \sim U_{0}\right)$, only the direct two-particle tunnelling is important, competing with second-order pair tunnelling. In fact, let us assume as an initial condition $c_{n, m}(0)=A_{n}(0) \delta_{n, m}$, that is, the two particles are initially placed on the same site, with a probability $\left|A_{n}(0)\right|^{2}$ to find both particles at site $n$. Then an asymptotic analysis of the underlying equations shows that the two particles form a bound state, that is they co-tunnel along the lattice, undergoing $\mathrm{BO}$ at the frequency $2 \omega_{\mathrm{B}}=2 F d$ twice the single-particle BO frequency $\omega_{\mathrm{B}}$. In Fock space, this means that we expect to observe, along the main diagonal $m=n$ of the square lattice of Fig. 1e, periodic revivals at frequency $2 \omega_{\mathrm{B}}$. Precisely, as shown in the Supplementary Note 2 (see also the Supplementary Figs S1 and S2), the bound-particle occupation amplitudes $A_{n}(t) \sim c_{n, n}(t)$ at the various lattice sites evolve according to equation (2), but with $F$ replaced by $2 F$ and $\kappa$ replaced by $\kappa_{\text {eff }}$. The effective hopping rate of the bound-particle state, $\quad \kappa_{\text {eff }}=-2 \kappa^{2} / U_{0}+\rho=-\varepsilon^{2} J^{2} /\left(2 U_{0}\right)-2 U_{0} \varepsilon^{2}, \quad$ includes second-order tunnelling $\left(-2 \kappa^{2} / U_{0}\right)$ and direct two-boson tunnelling $(\rho)$ processes. Such two contributions always add with the same sign, for both repulsive $\left(U_{0}>0\right)$ and attractive $\left(U_{0}<0\right)$ interactions, and generally are of the same order of magnitude. The bound-particle state thus behaves like a single particle hopping on the $1 \mathrm{D}$ lattice, but with a driving force which is doubled as compared with that of a single particle ${ }^{8,19}$ and with a modified hopping rate $\kappa_{\text {eff. }}$. Note that the effect of direct twoatom tunnelling on the $\mathrm{BO}$ dynamics, not considered in previous works ${ }^{19,20,22}$, is to increase the amplitude of the breathing motion (owing to the correction of $\kappa_{\text {eff }}$ ), whereas the periodicity of the $\mathrm{BO}$ is preserved.

Experimental photonic simulator. In our experiments, singleparticle hopping dynamics in the square lattice of Fig. 1e has been simulated by discretized spatial light transport in an engineered 2D square lattice of evanescently coupled optical waveguides ${ }^{29}$. This lattice has been fabricated in a fused silica substrate by direct waveguide writing with femtosecond lasers ${ }^{30-34}$, taking advantage of the 3D capabilities of this technology. The spatial propagation of the light intensity in the generic $(m, n)$-th lattice site maps the temporal evolution of the two-particle probability distribution $\left|c_{m, n}(t)\right|^{2}$ in Fock space ${ }^{29,33}$. In the photonic simulator of $\hat{H}_{\mathrm{EBH}}$, the role of the hopping coefficients $\kappa$ and $\rho$ is played by the evanescent coupling constants between first- and secondneighbouring waveguides $(\bar{\kappa}$ and $\bar{\rho})$, respectively. The former is in general greater than the latter and the relative weight can be tuned by properly engineering the lattice parameter $d$ (Supplementary Note 3 and Supplementary Fig. S3b,c). The on-site particle-interaction defect $U_{0}$ is realized by fabricating the main diagonal waveguides with a different refractive index change (this is achieved by slightly modifying the writing speed of the waveguides, as discussed in the Supplementary Note 3 and illustrated in the Supplementary Fig. S3a) and thus with the propagation constant $\beta^{\prime}$ slightly detuned with respect to that of all the others $(\beta)$. The positive or negative sign of the relative detuning $\Delta \beta=\beta-\beta^{\prime}$ determines the repulsive or attractive nature of the interaction, respectively. Lastly, we did not add any special feature in the nearest-neighbouring diagonals, because, as already discussed above, the nearest-site effects described in Fig. 1c are negligible with respect to the other terms of the EBH model. The implementation of the driving force term $\hat{H}_{F}$ is obtained by a constant bending of the waveguide axes (radius of curvature $R, F \propto 1 / R$ ) in the plane determined by the lattice main diagonal direction $Y$ and the light propagation direction $z$ (ref. 10). In Fig. 2a, the whole lattice structure is depicted. Note that the array cross-section is rotated in such a way that the bending plane is horizontal in the laboratory reference frame $\left(45^{\circ}\right.$ rotation with respect to Fig. 1e). This simplifies the fabrication process and the imaging of the light propagating in the waveguides; in this way, although being a $3 \mathrm{D}$ structure, each waveguide remains on a specific horizontal plane.

In a first experiment, we checked that our photonic simulator can reliably mimic the dynamics governed by $\hat{H}_{\mathrm{EBH}}$ alone, namely without the action of the external constant force. For this purpose, we fabricated 225 straight waveguides $(R=\infty)$ arranged in a $15 \times 15$ square lattice, with spacing $d=19 \mu \mathrm{m}$ and a length $L=2.5 \mathrm{~cm}$. For such a lattice spacing, the coupling coefficients are $\bar{\kappa}=0.95 \mathrm{~cm}^{-1}$ and $\bar{\rho}=0.3 \mathrm{~cm}^{-1}$. To simulate particle interaction, we fabricated the main diagonal waveguides with a propagation constant detuning $\Delta \beta=-4 \mathrm{~cm}^{-1}$, therefore representing an attractive interaction between the particles. A microscopic image of the array section is reported in Fig. 2b. We coupled $633 \mathrm{~nm} \mathrm{He-Ne}$ laser radiation into the central waveguide, belonging to the main diagonal subset and thus corresponding to an initial condition where the two particles occupy the same lattice site. As previously discussed, light is expected to preferably remain on the detuned diagonal, representing the fact that the correlated particles will hop together to the next site and will hardly separate. To study the evolution of the system, we monitored the light propagation along the array by imaging from above the fluorescence light at $650 \mathrm{~nm}$ emitted from the waveguides and arising from colour centres created in the femtosecond laser-writing process. As the colour centres are formed exclusively inside the waveguides, this technique yields a high signal-to-noise ratio and permits a direct imaging of the light propagation in the waveguide arrays ${ }^{33,34}$. The result obtained in this first experiment is visible in Fig. 3a. The

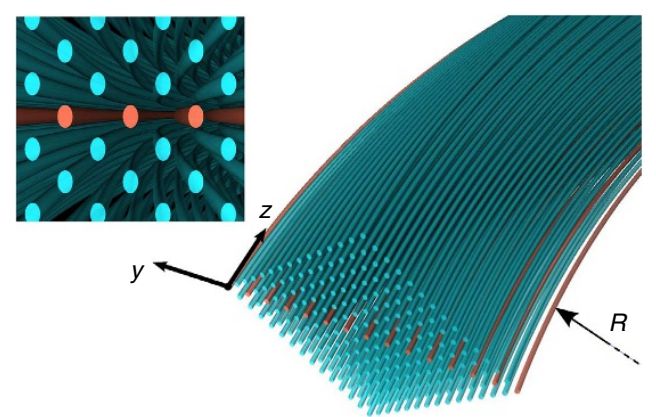

b

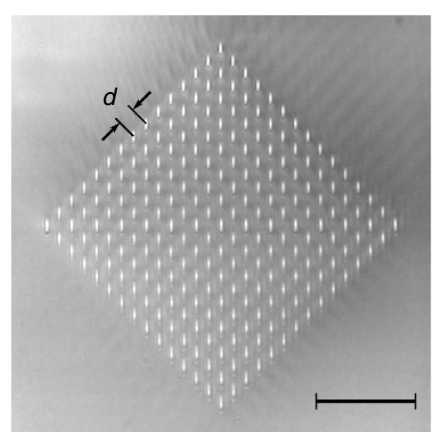

Figure 2 | The photonic simulator of correlated BO. (a) Sketch of the waveguide array structure; red-coloured waveguides have a different refractive index change with respect to the others to implement the on-site particle-interaction defect $U_{0}$. (b) Section of the fabricated array, imaged with an optical microscope. Scale bar, $100 \mu \mathrm{m}$. 


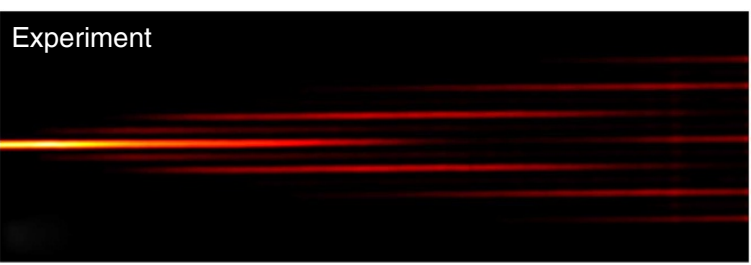

b

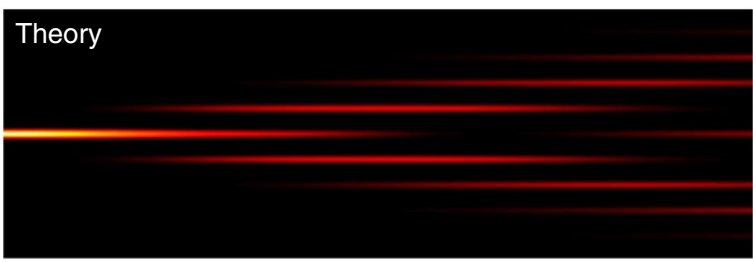

C
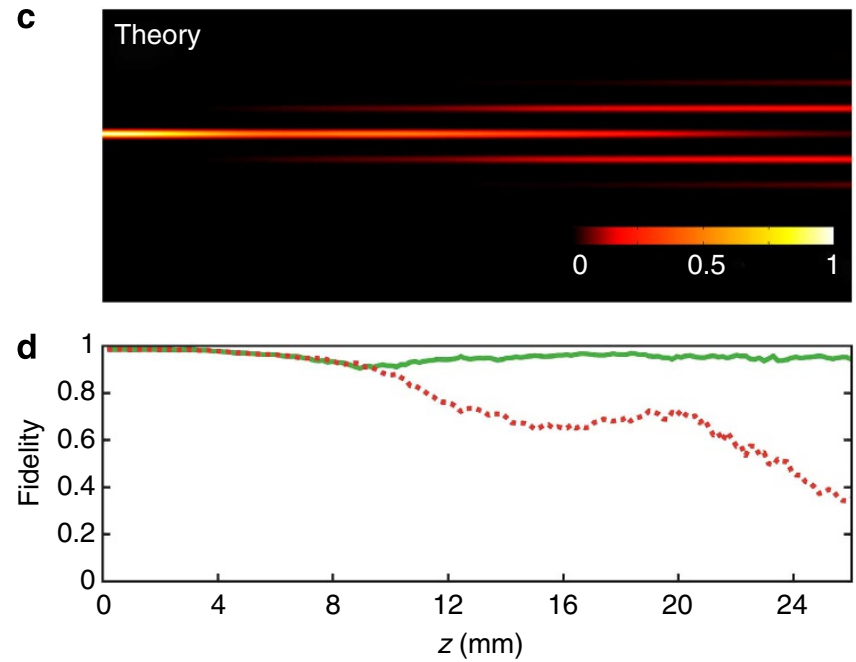

Figure 3 | Photonic simulation of the delocalization dynamics of two interacting particles in a lattice. (a) Experimental simulation in a waveguide lattice implementing direct two-particle tunnelling and secondorder pair tunnelling with a similar weight. (b) Numerical simulation including both contributions (EBH model). (c) Numerical simulation including only second-order pair tunnelling (BH model). (d) Fidelity (see main text for definition) of the experimental light distribution with that predicted by the $\mathrm{EBH}$ model (continuous line) and with that predicted by the standard $\mathrm{BH}$ model (dotted line)

measured distribution of light in the photonic model implementing both contributions to the bound state hopping rate, that is, twoparticle direct tunnelling and second-order pair tunnelling, is compared with the corresponding numerical simulation (Fig. 3b). The excellent agreement demonstrates that our photonic model can indeed take into account both contributions and thus correctly implements the EBH model. The role of the direct two-particle tunnelling (Fig. 1d), neglected in the standard $\mathrm{BH}$ model, can be quite relevant as shown by the different light distributions obtained from numerical simulations of the same structure but including only the second-order tunnelling (Fig. 3c).

To introduce a more quantitative comparison between the experimental light distribution in the array with the expected one, we introduce a metric, called fidelity, which provides the overlap between the experimental, $I_{\exp }$, and theoretical, $I_{\text {theo }}$, intensity profiles at each position $z$. Such metric is analytically defined as

$$
F_{z}=\frac{\int_{\Delta Y_{z}} I_{\exp } I_{\text {theo }} \mathrm{d} Y}{\sqrt{\int_{\Delta Y_{z}} I_{\exp }^{2} \mathrm{~d} Y \cdot \int_{\Delta Y_{z}} I_{\text {theo }}^{2} \mathrm{~d} Y}}
$$

where $\Delta Y_{z}$ indicates, at a given propagation distance $z$, the transverse coordinate interval $\Delta Y$ along which the intensity profile is considered. This metric ranges between 0 for completely uncorrelated profiles to 1 for perfect overlapping. We have calculated this function for the graphs in Fig. 3a-c and reported the results in Fig. 3d. Also from the quantitative point of view, it can be concluded that our photonic simulator is capable of faithfully implementing the EBH model, with an average fidelity as high as $95 \%$. In addition, it can be observed how modelling the same phenomenon with a standard $\mathrm{BH}$ model provides quite different predictions already after a limited propagation length with a fidelity to the experimental results as low as $35 \%$ at the end of the device.

A careful observation of Fig. 3a would reveal the presence of illuminated waveguides at interstitial positions with respect to the waveguide array at the detuned diagonal plane. These waveguides are actually at a different depth and belong to the first neighbouring plane. Their faint illumination (partially due to an out-of-focus condition) is nevertheless a clear indication that, due to the second-order pair tunnelling mechanism, light spreads also outside the main diagonal plane.

Observation of fractional BO. In a second experiment, we proceeded to the observation of fractional $\mathrm{BO}$. To this purpose, we fabricated another $15 \times 15$ square waveguide lattice, with the same lattice spacing and detuning on the diagonal as in the previous structure. To mimic the external force $F$, the waveguides are now circularly bent with a radius $R=400 \mathrm{~cm}$, as depicted in Fig. 2a. The array length is $L=8.5 \mathrm{~cm}$. As in the previous experiment, we injected the probe light into the central waveguide and we imaged its propagation along the waveguide lattice diagonal (Y), where light is confined. Figure $4 \mathrm{a}$ shows the oscillatory behaviour of the light dynamics along the waveguide array. Initially, light spreads into several waveguides, until it reaches a maximum of the breathing amplitude (around $L_{2, \max }=3.25 \mathrm{~cm}$ ). Then light refocuses into the central waveguide $\left(L_{2, \mathrm{foc}}=6.5 \mathrm{~cm}\right)$ and the breathing starts again. This periodic behaviour represents the correlated $\mathrm{BO}$ of two interacting particles, watched in Fock space. The accuracy of the fabricated photonic simulator is confirmed by the very good agreement with the numerical simulations of light propagation in the designed structure (Fig. 4b). A quantitative estimation of the agreement is provided by the fidelity, reported in Fig. 4c, that yields an average value of $90 \%$. To better analyse this result, we cut the sample at $L_{2, \max }$ and $L_{2, \text { foc }}$ and we imaged onto a CCD camera the light distribution exiting the output facet of the array (Fig. 5). Figure 5a clearly shows that, even in correspondence of the breath maximum amplitude, light remains mainly confined in the waveguide lattice-detuned diagonal. This fact is a further demonstration that two interacting particles, initially in the same lattice site, form a bound state and hop together to neighbouring sites. Figure $5 \mathrm{~b}$ shows that at the refocusing distance, all the light is indeed confined in the central waveguide, which was the one originally excited (indicated by a white arrow in both panels). A numerical simulation of the end-view distribution of light in the $2 \mathrm{D}$ array along the whole propagation length (Supplementary Movie 1) confirms the experimentally observed pattern.

The 'fractional' nature of $\mathrm{BO}$ for correlated particles can be appreciated by comparing Fig. 4a with the BO pattern for singleparticle hopping in the same lattice under the action of the same force $F$, shown in Fig. 4d. We fabricated a planar 1D array of 23 identical waveguides, equally spaced of $d^{\prime}=d=19 \mu \mathrm{m}$, and uniformly bent in the array plane with a constant radius of curvature $R^{\prime}$. Such a structure reliably simulates the dynamics of single particle $\mathrm{BO}^{10}$. To implement the same force on the single particle, we have to project the curvature on the diagonal for the two-particles structure on one of the main axis of the square 
a

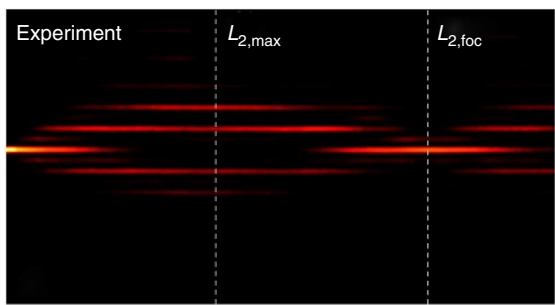

b
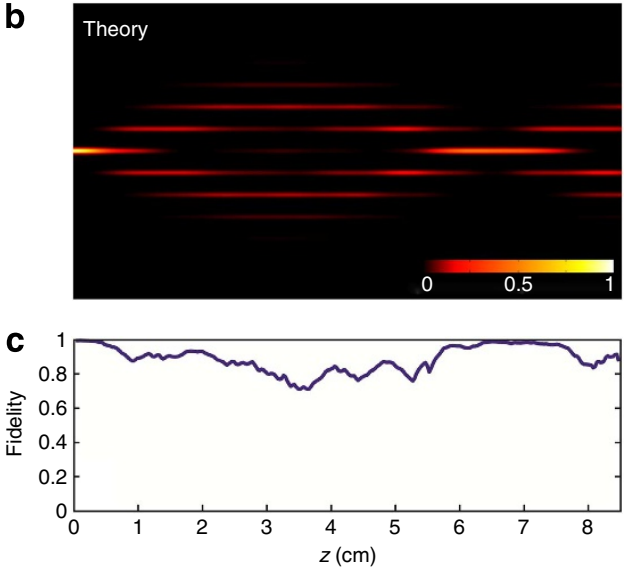

d

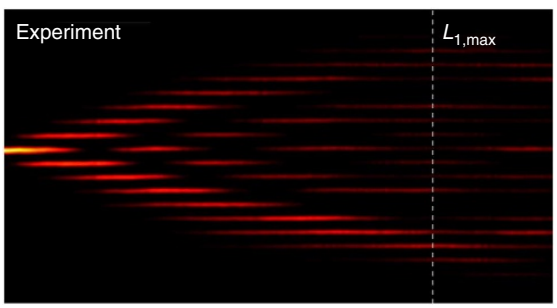

e
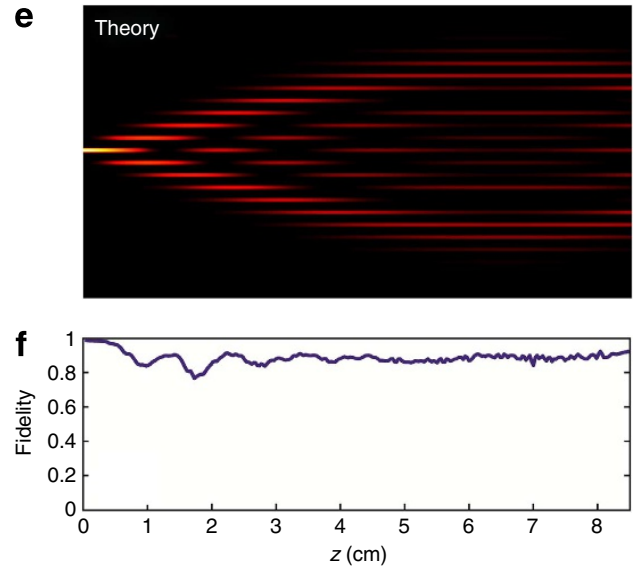

Figure 4 | Photonic simulation of BO for correlated and single particles. (a) Experimental and (b) numerical images of the light intensity distribution in the waveguide lattice main diagonal versus propagation distance $z$, representing in Fock space the BO for two interacting particles. In $\mathbf{c}$, the evolution of the fidelity, as given by equation (3), is also shown. (d-f): same as (a-c), but for the single-particle BO in the 1D waveguide array. Note that in this case, the position where the $\mathrm{BO}$ amplitude is at maximum $\left(L_{1, \max }\right)$ corresponds to the refocusing condition for the two-particle $B O\left(L_{2, \text { foc }}=L_{1, \text { max }}=6.5 \mathrm{~cm}\right)$.

a

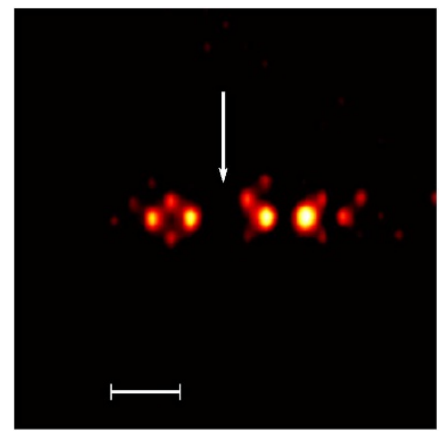

b

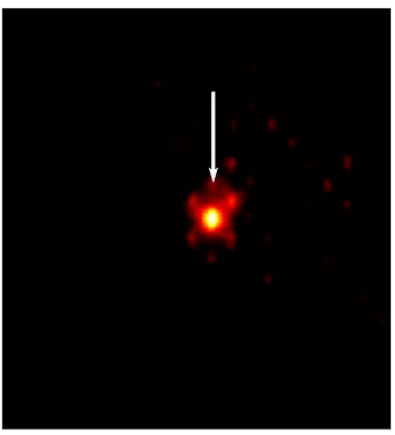

Figure 5 | Output light distribution at two propagation lengths.

Measured light distribution in the waveguide lattice section (corresponding to Fig. $4 a$ ) at positions (a) $L_{2 \text {,max }}$ where the fractional $\mathrm{BO}$ amplitude is at maximum and (b) $L_{2, \text { foc }}$ where light refocuses to the initial waveguide (indicated by a white arrow in both panels). Scale bar, $50 \mu \mathrm{m}$.

lattice: this yields a curvature radius for the linear array $R^{\prime}=R \sqrt{2} \simeq 566 \mathrm{~cm}$. We imaged the light propagation in this waveguide array for a length $L=8.5 \mathrm{~cm}$ (Fig. $4 \mathrm{~d}$ ). Also in this case, we observe a breathing propagation pattern corresponding to the single-particle $\mathrm{BO}$, but the position of the maximum breath amplitude $L_{1, \max }$ corresponds to the refocusing position $L_{2, \text { foc }}$ for two-particles BO (Fig. 4a). This demonstrates that the frequency of a two-particles BO is twice that for a single-particle. It may be worth noting that the breathing amplitude of a single-particle $\mathrm{BO}$ is larger than that for the two-particle BO. This is due to a higher hopping rate in the former case, $\kappa>\kappa_{\text {eff }}$, and to the double force experienced by the two strongly correlated particles with respect to the single one. The agreement between the experimental light distribution (Fig. 4d) and the theoretical one (Fig. 4e) is reported in Fig. $4 \mathrm{f}$ in terms of fidelity, yielding an average value of $90 \%$.

\section{Discussion}

We have experimentally observed the photonic quantum analogy of fractional BO of two strongly correlated particles. The Fock space dynamics of the two-particle EBH Hamiltonian is mapped into spatial propagation of discretized light in an engineered square lattice of evanescently coupled optical waveguides. We have experimentally verified the doubling of the $\mathrm{BO}$ frequency of correlated particles with respect to that observed for a single particle, and shown the competition of second-order tunnelling and direct two-particle tunnelling. The simple visualization of the phenomenon and the high control of the fabricated structure make our photonic model system a very powerful tool to investigate exotic non-equilibrium phenomena associated to the formation of particle-bound states that are difficult to access or prepare in the matter. It is envisaged that our model system could offer the possibility to visualize other intriguing phenomena involving few correlated particles, such as the interplay between particle interaction and Anderson localization 35,36 , quantum billiards and transition to quantum chaos $^{23}$, anyonic $\mathrm{BO}^{37}$, correlated barrier tunnelling and particle dissociation $^{38}$.

\section{Methods}

Fabrication of the waveguide arrays. Waveguide arrays have been fabricated by femtosecond laser waveguide writing in fused silica samples. The second harmonic of a HighQLaser femtoREGEN Yb-based amplified laser system has been used, consisting in 400 -fs pulses at 520 -nm wavelength with repetition rates up to $960 \mathrm{kHz}$. Actual writing conditions consisted of 300-nJ pulses delivered at a repetition rate of $20 \mathrm{kHz}$. Optimal range for the writing speed was identified as $8-14 \mathrm{~mm} \mathrm{~s}^{-1}$, yielding propagation losses of about $0.5 \mathrm{~dB} \mathrm{~cm}^{-1}$. To implement the detuning in the lattice diagonal, we modulated the writing speed, therefore the waveguides on the diagonal were fabricated at a speed of $9 \mathrm{~mm} \mathrm{~s}^{-1}$, while all the others were written at $14 \mathrm{~mm} \mathrm{~s}^{-1}$. The variation in writing speed modifies the propagation constants of the guided modes, producing the designed detuning $\Delta \beta=-4 \mathrm{~cm}^{-1}$, but has a negligible effect on the coupling rate between the waveguides. End faces of the substrates were polished after laser writing to improve light launching and output imaging. 
Characterization of the light distribution in the arrays. Femtosecond laser writing in fused silica creates colour centres that provide fluorescent emission at about $650 \mathrm{~nm}$, when light at $633 \mathrm{~nm}$ is propagated in the waveguide. Top-view imaging of the fluorescence signal is employed to visualize and quantitatively estimate the light distribution along the waveguide array, rejecting the background light by a notch filter at $633 \mathrm{~nm}$. Light collection for the top-view images is performed with a $\times 10$ objective, 0.25 numerical aperture, placing the detuned diagonal at focus. The waveguides in the neighbouring planes go progressively out of focus, as the distance between planes is $13.5 \mu \mathrm{m}$ and the Rayleigh range of the collecting optics is $z_{\mathrm{R}}=4.6 \mu \mathrm{m}$; however, the first neighbouring plane of waveguides is still faintly visible in some of the images. To achieve a high resolution in imaging the light in the waveguide array all along its length, several images have been acquired and then stitched together. Propagation losses in the waveguides have been compensated by renormalizing the intensity levels in the acquired images.

\section{References}

1. Bloch, F. Über die Quantenmechanik der Electronen in Kristallgittern. Z. Phys. 52, 555-600 (1928).

2. Zener, C. A theory of electrical breakdown of solid dielectrics. R. Soc. Lond. A 145, 523-529 (1934).

3. Waschke, C. et al. Coherent submillimeter-wave emission from Bloch oscillations in a semiconductor superlattice. Phys. Rev. Lett. 70, 3319-3322 (1993).

4. Ben Dahan, M., Peik, E., Reichel, J., Castin, Y. \& Salomon, C. Bloch oscillations of atoms in an optical potential. Phys. Rev. Lett. 76, 4508-4511 (1996).

5. Wilkinson, S. R., Bharucha, C. F., Madison, K. W., Niu, Q. \& Raizen, M. G. Observation of atomic Wannier-Stark ladders in an accelerating optical potential. Phys. Rev. Lett. 76, 4512-4515 (1996).

6. Anderson, B. P. \& Kasevich, M. A. Macroscopic quantum interference from atomic tunnel arrays. Science 282, 1686-1689 (1998).

7. Morandotti, R., Peschel, U., Aitchison, J. S., Eisenberg, H. S. \& Silberberg, Y Experimental observation of linear and nonlinear optical Bloch oscillations. Phys. Rev. Lett. 83, 4756-4759 (1999).

8. Pertsch, T., Dannberg, P., Elflein, W., Bräuer, A. \& Lederer, F. Optical Bloch oscillations in temperature tuned waveguide arrays. Phys. Rev. Lett. 83, 4752-4755 (1999).

9. Sapienza, R. et al. Optical analogue of electronic Bloch oscillations. Phys. Rev. Lett. 91, 263902 (2003).

10. Chiodo, N. et al. Imaging of Bloch oscillations in erbium-doped curved waveguide arrays. Opt. Lett. 31, 1651-1653 (2006).

11. Trompeter, H. et al. Bloch oscillations and Zener tunneling in two-dimensional photonic lattices. Phys. Rev. Lett. 96, 053903 (2006).

12. Sanchis-Alepuz, H., Kosevich, Y. A. \& Sanchez-Dehesa, J. Acoustic analogue of electronic Bloch oscillations and resonant Zener tunneling in ultrasonic superlattices. Phys. Rev. Lett. 98, 134301 (2007).

13. Winkler, K. et al. Repulsively bound atom pairs in an optical lattice. Nature 441, 853-856 (2006).

14. Fölling, S. et al. Direct observation of second-order atom tunnelling. Nature 448, 1029-1032 (2007).

15. Buchleitner, A. \& Kolovsky, A. R. Interaction-induced decoherence of atomic Bloch oscillations. Phys. Rev. Lett. 91, 253002 (2003).

16. Gustavsson, M. et al. Control of interaction-induced dephasing of Bloch oscillations. Phys. Rev. Lett. 100, 080404 (2008).

17. Eckstein, M. \& Werner, P. Damping of Bloch oscillations in the Hubbard model. Phys. Rev. Lett. 107, 186406 (2011).

18. Claro, F., Weisz, J. F. \& Curilef, S. Interaction-induced oscillations in correlated electron transport. Phys. Rev. B 67, 193101 (2003).

19. Dias, W. S., Nascimento, E. M., Lyra, M. L. \& de Moura, F. A. B. F. Frequency doubling of Bloch oscillations for interacting electrons in a static electric field. Phys. Rev. B 76, 155124 (2007).

20. Khomeriki, R., Krimer, O., Haque, M. \& Flach, S. Interaction-induced fractional Bloch and tunneling oscillations. Phys. Rev. A 81, 065601 (2010).
21. Bloch, I., Dalibard, J. \& Nascimbene, S. Quantum simulations with ultracold quantum gases. Nature Phys. 8, 267-276 (2012).

22. Longhi, S. Photonic Bloch oscillations of correlated particles. Opt. Lett. 36, 3248-3250 (2011)

23. Krimer, D. O. \& Khomeriki, R. Realization of discrete quantum billiards in a two-dimensional optical lattice. Phys. Rev. A 84, 041807 (R) (2011).

24. Mazzarella, G., Giampaolo, S. M. \& Illuminati, F. Extended Bose-Hubbard model of interacting bosonic atoms in optical lattices: from superfluidity to density waves. Phys. Rev. A 73, 013625 (2006).

25. Liang, J. Q., Liu, J. -L., Li, W. -D. \& Li, Z. -J. Atom-pair tunneling and quantum phase transition in the strong-interaction regime. Phys. Rev. A 79, 033617 (2009).

26. Trotzky, S. et al. Time-resolved observation and control of superexchange interactions with ultracold atoms in optical lattices. Science 319, 295-299 (2008).

27. Mendez, E. E. \& Bastard, G. Wannier-Stark ladders and Bloch oscillations in superlattices. Phys. Today 46, 34-42 (1993).

28. Gluck, M., Kolovsky, A. R. \& Korsch, H. J. Wannier-Stark resonances in optical and semiconductor superlattices. Phys. Rep. 366, 103-182 (2002).

29. Christodoulides, D., Lederer, F. \& Silberberg, Y. Discretizing light behavious in linear and nonlinear waveguide lattices. Nature 424, 817-823 (2003).

30. Osellame, R., Cerullo, G. \& Ramponi, R. (eds). Femtosecond Laser Micromachining: Photonic and Microfluidic Devices in Transparent Materials, Topics in Applied Physics Vol. 123 (Springer, 2012).

31. Gattass, R. R. \& Mazur, E. Femtosecond laser micromachining in transparent materials. Nature Photon. 2, 219-225 (2008).

32. Crespi, A., Longhi, S. \& Osellame, R. Photonic realization of the quantum Rabi model. Phys. Rev. Lett. 108, 163601 (2012).

33. Szameit, A. \& Nolte, S. Discrete optics in femtosecond-laser-written photonic structures. J. Phys. B At. Mol. Opt. Phys. 43, 163001 (2010).

34. Szameit, A. et al. Polychromatic dynamic localization in curved photonic lattices. Nature Phys. 5, 271-275 (2009).

35. Krimer, D. O., Khomeriki, R. \& Flach, S. Two interacting particles in a random potential. JETP Lett. 94, 406-412 (2011).

36. Albrecht, C. \& Wimberger, S. Induced delocalization by correlation and interaction in the one-dimensional Anderson model. Phys. Rev. B 85, 045107 (2012).

37. Longhi, S. \& Della Valle, G. Anyonic Bloch oscillations. Phys. Rev. B 85, 165144 (2012)

38. Kolovsky, A. R., Link, J. \& Wimberger, S. Energetically constrained co-tunnelling of cold atoms. New J. Phys. 14, 075002 (2012).

\section{Acknowledgements}

This work was supported by the European Union through the project FP7-ICT-2011-9600838 (QWAD -Quantum Waveguides Application and Development).

\section{Author contributions}

All authors conceived the experiment. G.C., A.C., R.O. designed and fabricated the photonic device and performed the measurements. G.D.V. and S.L. developed the theory underlying the experiment. All authors discussed the results and participated in the manuscript preparation.

\section{Additional information}

Supplementary Information accompanies this paper at http://www.nature.com/ naturecommunications

Competing financial interests: The authors declare no competing financial interests

Reprints and permission information is available online at http://npg.nature.com/ reprintsandpermissions/

How to cite this article: Corrielli, G. et al. Fractional Bloch oscillations in photonic lattices. Nat. Commun. 4:1555 doi: 10.1038/ncomms2578 (2013). 